\title{
Chapter 43. Intracranial electrophysiology in language research
}

\author{
Adeen Flinker, Vitória Piai, and Robert T. Knight
}

\subsection{Introduction}

Intracranial electrophysiological recording in humans has been a long standing technique in neurosurgical treatment for epilepsy and have served as an important window in to how the human brain processes language. This chapter is aimed to introduce the reader to the technique, how it historically contributed to language mapping, its advantages and disadvantages as a research tool, and analysis techniques that have provided novel findings and approaches in the area of language processing.

\subsection{History of epilepsy surgery}

The pioneering work of Paul Broca and Carl Wernicke localizing speech deficits to the inferior frontal gyrus (IFG) (Broca, 1861) and posterior temporal lobe (Wernicke, 1874) sparked a new era in the neurobiology of language, as well as greatly influencing subsequent approaches to neurosurgical treatment of epilepsy. Broca's observation together with the finding that electrical stimulation of cortex can produce specific motor responses (Hitzig, 1900) paved the road to consider epilepsy a form of irritated tissue and treatable by resection of the disruptive tissue (Feindel et al., 2009). Cortical resection became a safe and effective treatment for focal epilepsy based on stimulation mapping of motor function as well as epileptic foci (Horsley, 1909; Krause \& Thorek, 1912). These stimulation techniques were augmented after the discovery of Electroencephalography (EEG) by Hans Berger (Berger, 1929; 1931) and its acceptance by the international community (Adrian \& Matthews, 1934; Feindel et al., 2009). EEG proved to be a powerful tool in the classification and diagnosis of epileptic activity (Gibbs et al., 1936; Jasper \& Kershman, 1941) and was first recorded intraoperatively in humans under local anesthesia while subjects were awake and stimulation mapping was performed (Feindel et al., 2009; Foerster \& Altenburger, 1935). Electrocorticography (ECoG) or intracranial EEG (iEEG) soon became a routine technique in aiding neurosurgeons during cortical mapping (Jasper et al., 1951; Penfield \& Erickson, 1941). During these early surgeries stimulation mapping provided great insight into the topo graphic organization of motor and somatosensory cortices culminating in the famous cortical homunculus diagram (Penfield \& Boldrey, 1937). While mapping of motor cortex was prevalent since the turn of the twentieth century (Cushing, 1909), Penfield and colleagues in the Montreal Neurological Institute (MNI) were the first to systematically extend stimulation mapping to language and devised protocols that remain largely unchanged to this day (Penfield \& Roberts, 1959). During a typical electrical stimulation mapping (ESM) procedure, the patient is awakened from anesthesia while cortex is still exposed and is asked to perform the same tasks Penfield first employed over 80 years ago: counting and picture naming. While the patient engages in counting or picture naming, different cortical sites are repeatedly stimulated. If the applied electrical current reliably disrupts the patient's speech output, or causes naming errors with intact speech output, the cortical site is deemed to be critical for language and is spared from resection. ESM has been invaluable in sparing neo cortical sites critical for language, but these sites have been found over distributed regions of frontal, temporal, and parietal cortex and with a high degree of intersubject variability (Ojemann et al., 1989; Sanai et al., 2008). Even though counting and picture naming tasks tap into a relatively small subset of language functions, sparing of sites identified by cortical stimulation dramatically reduces postoperative deficits and the procedure remains the gold standard in the field (; Chang et al., 2014; Sanai et al., 2008).

\subsection{Intracranial EEG monitoring}

ESM and definition of the epileptogenic zone are mostly performed in the operating room, as pioneered by Penfield and colleagues. However, this constrains the time available for testing and monitoring the patients. In cases where the epileptic zone is unclear, patients are implanted with an array of electrodes for a period 
spanning several days to weeks (Ojemann, 1987; Wieser \& Elger, 2012). During this time, intracranial EEG is recorded from the electrodes to more clearly define epileptic zones as well as map function via electrical stimulation. After clinical assessment has completed, the electrodes are removed, and epileptic tissue is resected. In addition to providing a more refined mapping of cognitive function for clinical purposes, the extended time these patients spend in the monitoring unit provides ample opportunity to engage with patients in research studies (Crone, 2000). During lulls in clinical treatment, patients may consent to different cognitive and language tasks at their convenience in a bedside setting. The acquisition of electrical signals directly from cortex provides a valuable opportunity to explore language function and while electrode locations are solely guided by the clinical necessity of the patient, they often sample perisylvian regions. During the late 1990s there was a surge of research performed in the chronic bedside setting. The most common procedure in North America is the implantation of subdural grids and strips, referred to as electrocorticography $(\mathrm{ECoG})$, which provide a wide spatial coverage on the surface of cortex but are typically limited to one hemisphere and the surface of cortex surrounding the area of the craniotomy (Gonzalez Martinez et al., 2013; Risinger \& Gumnit, 1995; Widdess Walsh et al., 2007). In cases where deeper structures, such as the hippocampus, amygdala, orbitofrontal cortex, or insula, need to be monitored for epileptic activity depth electrodes with several contacts are implanted as well (Ojemann, 1987). An alternative approach was developed in the Paris school of Talairach and colleagues whereby depth electrodes are implanted stereotactically, stereotactic EEG or sEEG, enabling the monitoring of epileptic activity across the depth of cortical structures in both hemispheres and is more widely adapted in Europe (Crandall \& Babb, 1993; Lüders, 2008; Talairach et al., 1958). Both have clinical advantages and disadvantages (Wellmer et al., 2012) and can be complementary approaches depending on the hypothesized epileptogenic zone (Mullin et al., 2016).

\subsection{Methodological considerations}

Due to the nature of epilepsy, that often involves temporal lobe structures, as well as the clinical necessity to map critical language sites prior to resection of tissue, electrode coverage often involves perisylvian cortices. This cortical sampling as well as the combined temporal and spatial resolution provided by intracranial recordings offer a unique opportunity to address language processing. Compared with noninvasive electrophysiology (EEG, MEG), intracranial recordings do not suffer from volume conduction outside of cortex providing a much higher signal to noise ratio, a spatial resolution limited only by the electrode spacing (typically $1 \mathrm{~cm}$; Lachaux et al., 2003) as well as a lower susceptibility to muscle and eye movement artifacts (Crone, 2000). Both sEEG and ECoG are largely immune to muscle artifacts from eye, jaw, lip and tongue making them ideal for speech production studies (Llorens et al., 2011) as well as research on reading. Nevertheless, contamination from eye movements has been reported especially in the vicinity of the temporal pole and orbitofrontal cortex (Ball et al., 2009; Jerbi et al., 2009; Kovach et al., 2011), requiring some care classifying electrodes that may be prone to the effect. While intracranial recordings provide an excellent combined temporal and spatial resolution which cannot be established by noninvasive electrophysiology (EEG, MEG) or neuroimaging (fMRI, PET) alone, they do hold several drawbacks. Typical ECoG coverage involves a grid of electrodes (typically $8 \times 8$ with $1 \mathrm{~cm}$ spacing) as well as depth electrodes and supplementary strips depending on the clinical necessity (Fig. 43.1, right). The insertion of these electrodes requires a craniotomy and is limited to one hemisphere (although some limited sampling of the other hemisphere may be provided by insertion of depth or strip electrode via burr holes). This limits the spatial sampling to mainly one hemisphere and constrains within subject approaches for hypotheses concerning function of both hemi spheres, such as hemispheric asymmetry. Conversely, in sEEG the typical coverage involves depth electrodes bilaterally (burr holes bilaterally without a craniotomy) but the sampling is sparse and limited to the number of depth probes inserted (Fig. 43.1, left). Lastly, intracranial research is most commonly recorded from patients with epilepsy that may have had some degree of cortical reorganization during development to compensate for epileptic tissue. To address this issue, it is important 
to remove all epileptic electrodes from research analysis and include multiple subjects with varying epileptic sources. Signals acquired directly from cortex in animal models allow for varying degree of invasiveness as well as insertion of electrode probes on multiple scales. In human intra cranial research the most common electrodes provide a sampling of several millimeters of cortex. The typical clinical electrode is $4.0 \mathrm{~mm}$ in diameter and has $2.3 \mathrm{~mm}$ exposed to cortex with $1 \mathrm{~cm}$ interelectrode spacing (smaller and higher density pediatric grids are also used with $0.4-0.5 \mathrm{~mm}$ interelectrode spacing). These electrodes record activity from large neuronal populations beneath the area of cortex exposed to the electrode (Wieser \& Elger, 2012). In some cases, small microwire electrodes $(20-50 \mu \mathrm{m}$ diameter) are used which produce a signal typically referred to as a Local Field Potential (LFP) - although the nomenclature is freely used to reflect various recording techniques from cortex (Buzsáki et al., 2012) and the extent to which LFP is spatially limited is still debated (Kajikawa \& Schroeder, 2011). The LFP signal is extremely rich with both broadband information (i.e., delta, theta, alpha, beta, gamma rhythms and high gamma activity $>70 \mathrm{~Hz}$ and up to 250 $\mathrm{Hz}$ ) as well as activity in the $\mathrm{kHz}$ range which can be used to extract multiunit activity and in some instances individual neurons (single unit activity). The use of microwire recordings is rare in language research although there have been several reports from anterior temporal cortex, including neuronal responses in the superior and middle temporal gyri to specific phonetic cues in auditory sentences (Creutzfeldt et al., 1989), spatially organized tonotopic responses in Heschel's gyrus (Howard et al., 1996a), robust tracking of compressed speech (Nourski et al., 2009), as well as visual word recognition in the ventral temporal lobe (Halgren et al., 2006; 2015). Microwire electrodes are typically part of a larger clinical depth electrode with microcontacts at the tip using a hybrid approach (Howard et al., 1996b).

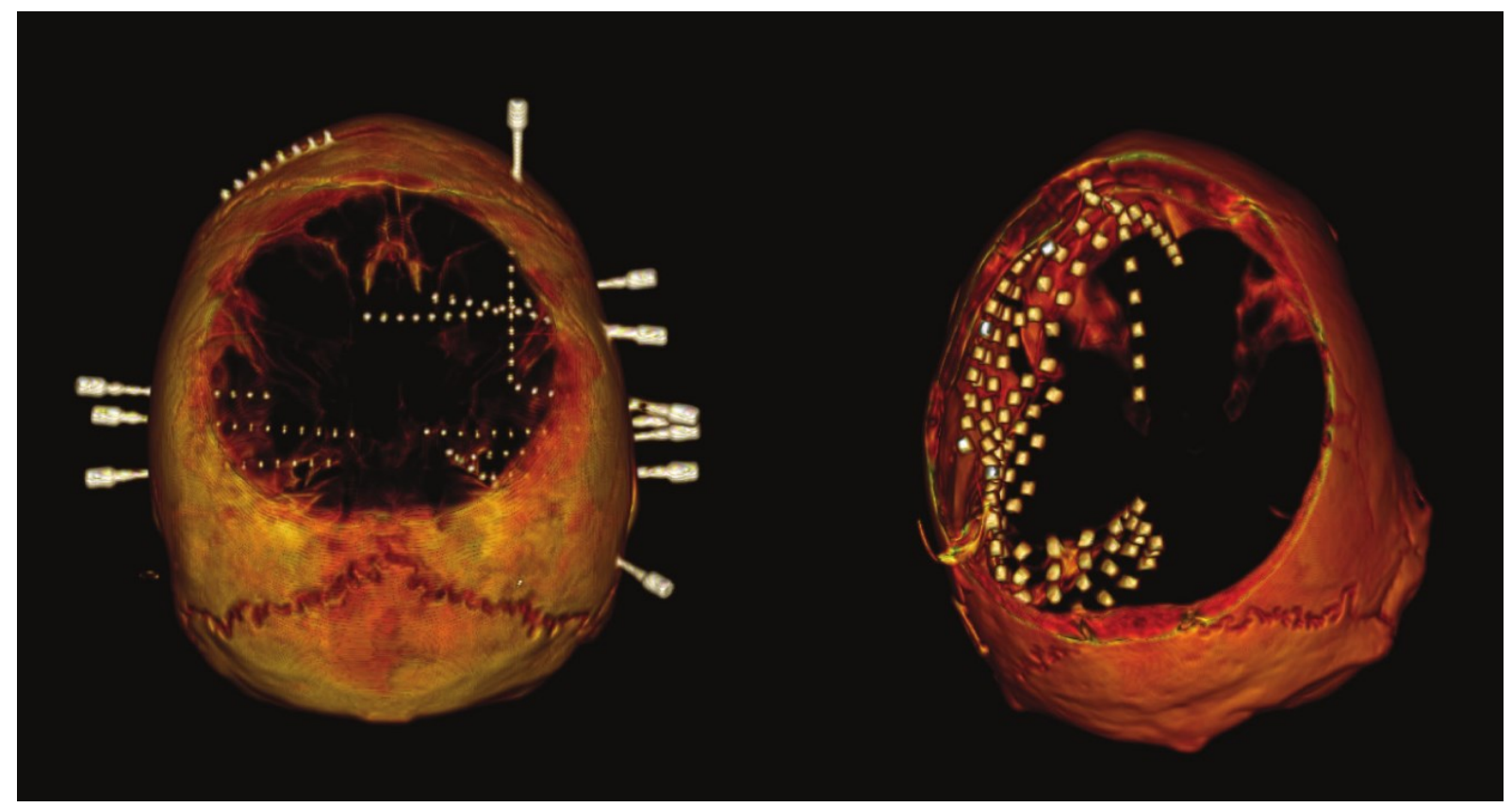

Fig. 43.1 sEEG and ECoG typical coverage. Superior view of 3D reconstructions of computed tomography (CT) scans in a sEEG (left) and ECoG (right) implantation of intracranial electrodes.

\subsection{Intracranial event related potentials}

Early intracranial recordings and analysis were limited to evoked potentials. Fried et al. recorded intraoperative event related potentials (ERP) during presentation of pictures. They found that premotor sites (likely IFG) showed a sustained negativity that was specific to a rhyming task compared with a visual angle discrimination task (Fried et al., 1981). In contrast to ECoG surface and depth recordings, sEEG provides routine coverage of both hemispheres. Liégeois Chauvel et al. localized early auditory evoked responses to specific regions of Heschel's gyrus (Liégeois Chauvel et al., 1991). Additionally, the authors 
provided direct electrophysiological evidence for a left hemisphere specificity to voice onset time (Liégeois Chauvel et al., 1999) and for a hemispheric difference in processing temporal modulations (Liégeois Chauvel et al., 2004). Similarly, deep brain regions such as the medial temporal lobe, and the hippocampus in particular, can only be reached with depth electrodes. Previous studies have focused on different research questions regarding the involvement of medial temporal lobe regions in language. McCarthy et al. tested patients reading correct sentences and sentences ending with a semantic violation (e.g., "I ordered a ham and cheese scissors.") (McCarthy et al., 1995). A negative deflection was observed in the ERPs for the semantic violations relative to the correct sentences, peaking around $400-500 \mathrm{~ms}$ post target word onset. This effect was focal, found in contacts in the anterior medial temporal lobe bilaterally. In another study (Nobre \& McCarthy, 1995), participants saw word(-like) stimuli presented individually on a screen. Their task was to press a button when a word was of a particular category (e.g., a body part). In one experiment, the stimuli presented consisted of different types of words: orthographically illegal non words (e.g., gtprlm), pseudowords (e.g., glubbalt), function words (e.g., hence), and content words (e.g., truck). In another experiment, all stimuli were content words that were paired as prime target words. Primes could either be semantically related or unrelated to the target words. Content words (including the primes) elicited a negative deflection in the ERPs peaking around $400 \mathrm{~ms}$ post stimulus onset in the anterior medial temporal lobe. The amplitude of this negative deflection decreased for pseudowords, and further decreased for function words and non-words. An attenuation of this negative deflection was also observed for semantic ally primed target words. In both studies, the N400 like potentials measured in contacts in the anterior medial temporal lobe likely reflects activity from the anterior fusiform and parahippocampal gyri, but not from the hippocampus proper. Meyer et al. had their participants read three types of sentences: correct sentences (e.g., "The door was being closed"), sentences containing semantic violations (e.g., "The ocean was being closed"), and sentences containing syntactic violations (i.e., a phrase structure violation, e.g., "The shop was being on closed") (Meyer et al., 2005). Recordings from the rhinal cortex revealed a negative deflection in the ERPs between 200 and 500 ms post target word onset for the correct and semantically incorrect sentences that was absent in the sentences with syntactic violations. By contrast, hippocampal recordings revealed a negative deflection between 500 and $800 \mathrm{~ms}$ for the syntactic violations that was absent in the other two types of sentences.

\subsection{Intracranial time frequency signatures}

In a series of ECoG papers Crone et al., investigated how different frequency bands can index and track cognitive function across a range of language (Crone et al., 1994; 2001a) and motor tasks (Crone et al., 1998a; 1998b). Alpha power decreases were found in three language production tasks, namely picture naming, word reading, and word repetition. These effects were found in electrodes over sensorimotor regions, superior temporal gyrus, and basal temporal occipital cortex. The early studies of Crone and colleagues focused on the alpha and beta frequency bands given their well-known relation to the sensorimotor cortex. In the memory domain, however, the theta band has been consistently linked to mnemonic processes in medial temporal lobe regions (Buzsáki \& Moser, 2013; Lega et al., 2012; Rutishauser et al., 2010). Based on this evidence, Piai and colleagues focused on the theta frequency band in the medial temporal lobe during a language task. They employed a sentence completion task in which participants named pictures that completed semantically constrained (e.g., "She swept the floor with a," [picture: BROOM]) or neutral sentences (Piai et al., 2016). Piai et al. observed increases in theta power for semantically constrained relative to neutral sentences during sentence processing, preceding picture presentation. Figure 43.2 shows this effect for 10 different patients. This effect was found not only in the hippocampus proper, but also in the parahippocampal gyrus and entorhinal cortex. These results provided evidence that medial temporal lobe structures contribute to language processing online, relating words in the sentence to stored semantic knowledge. Moreover, this process seems to be supported by the same neuronal computations performed by these structures for memory function, as reflected in theta oscillations. 


\subsection{Discovery of high gamma activity}

One of the key findings in the studies of Crone and colleagues was the existence of high gamma activity $(70-150 \mathrm{~Hz})$ that tracked cortical processing and provided a more robust and localized within subject index compared with low frequency desynchronization and ERPs (Crone, 2000; Crone et al., 2001b; 2006). While the extent to which high gamma power reflects band limited oscillations or broadband activity has been debated, it has been successfully employed across cognitive domains to track cortical activity (Jacobs et al., 2010; Crone et al., 2011; Miller, 2010). High gamma activity has been shown to be correlated with the spiking rate of underlying neurons as well as coupled to the hemodynamic BOLD response in both animal (Allen et al., 2007; Belitski et al., 2008; Ray et al., 2008) and human cortex (Mukamel et al., 2005; Nir et al., 2007). These findings have been followed by a growth of intracranial research elucidating cognitive function while leveraging this high frequency band (HFB) signal which is hard to detect outside the skull. The high frequency signal (referred to as high gamma, HFB and sometimes broadband signal) has become one of the most common cortical indices used to map cognitive function as well as track the perception and production of language. 

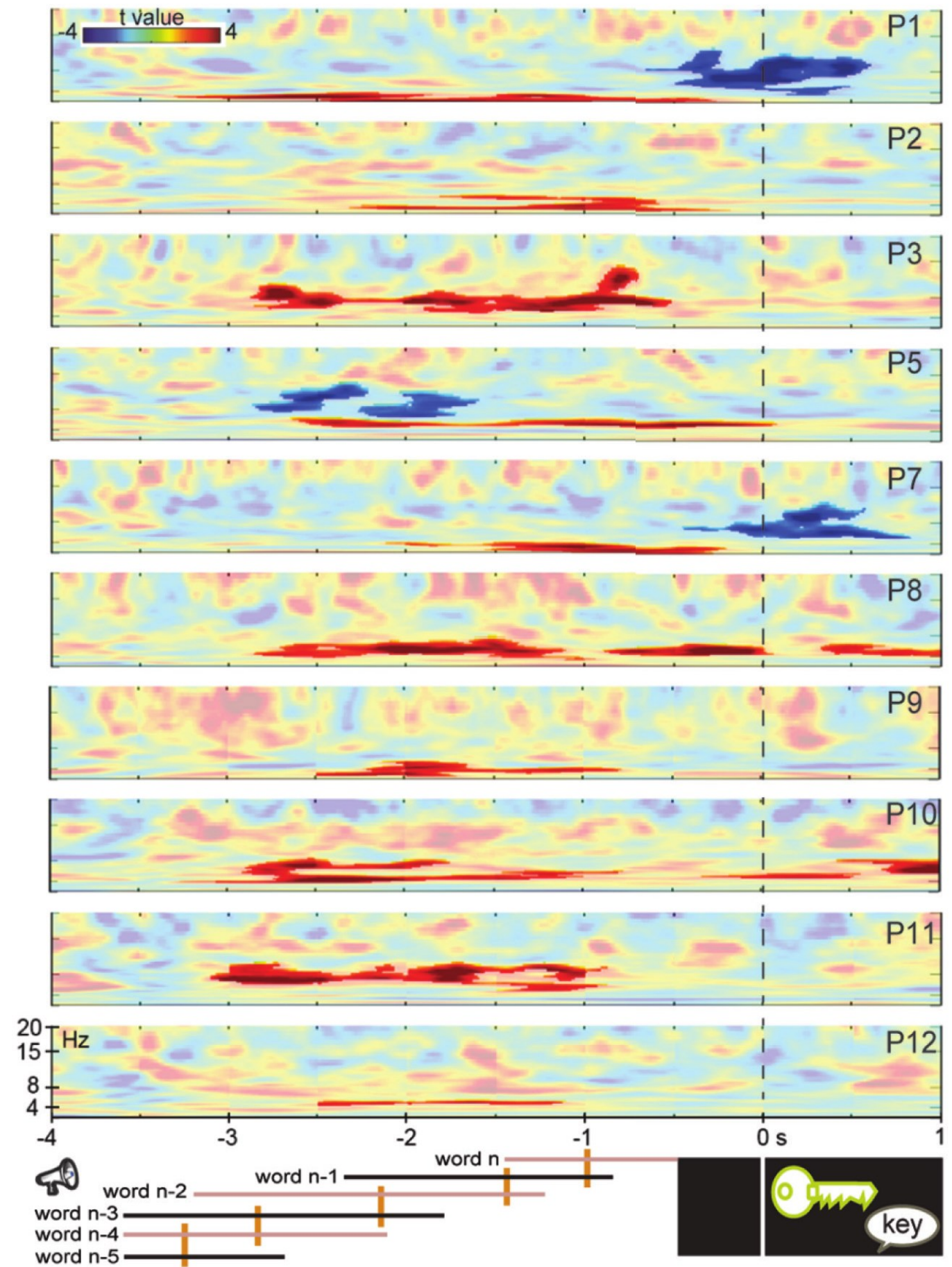

Fig. 43.2 Context effect (constrained vs. neutral) time locked to picture presentation. Significant theta effect is shown in stronger colors (multiple comparisons corrected). Trial events are shown at the bottom. The timing of each word position is indicated by the continuous lines. The left end of each line indicates the earliest possible word onset. The right end indicates the latest possible word offset (and next word onset). Median word onset (and previous word offset) is indicated by the orange vertical bars.

Adapted from Vitória Piai, Kristopher L. Anderson, Jack J. Lin, Callum Dewar, Josef Parvizi, Nina F. Dronkers, and Robert T. Knight, Direct brain recordings reveal hippocampal rhythm underpinnings of language processing, Proceedings
of the National Academy of Sciences of the United States of America, 113 (40), pp. 11366- 71, Figure 3, doi: 10.1073/ pnas.1603312113 O2016 Vitória Piai, Kristopher L. Anderson, Jack J. Lin, Callum Dewar, Josef Parvizi, Nina F. Dronkers,

The high gamma signal allows for a unique window into language function as it provides a signature for neural activity that is robust on the level of single trials with high temporal resolution as well as activation signatures similar to fMRI. In non-invasive electrophysiology, the high gamma signal is virtually inaccessible due to volume conductance effects and a sharp drop in power in higher frequencies when passing the skull. Furthermore, unlike neuroimaging and noninvasive electrophysiology, the position of electrodes directly on 
(or in) the brain renders intracranial signals minimally contaminated by motor artifacts from speech production or movement. Using a picture naming task and sEEG recordings from the medial temporal lobe, Hamamé et al., observed increases in high gamma power between picture presentation and initial articulation in the hippocampus (Hamamé et al., 2014). The peak latency of the high gamma activity correlated with the participants' picture naming latencies. Finally, tip of the tongue states (i.e., when a speaker recognizes an object but cannot retrieve its name) were associated with no increases in hippocampal high gamma activity relative to a pre stimulus baseline. Recordings from the surface of the temporal cortex have been mostly focused on acoustic, phonetic, and lexical levels of processing. Studies have established strong high gamma responses to auditory stimulation in the superior temporal gyrus (STG) when presented with non-speech stimuli (Crone et al., 2001a; Brugge et al., 2009; Edwards, 2005), phonemes (Chang et al., 2011; Crone et al., 2001a; Flinker et al., 2010; Fukuda et al., 2010) and words (Brown et al., 2008; Canolty et al., 2007; Crone et al., 2001b; Edwards et al., 2010; Flinker et al., 2011; Pei et al., 2011). Sites on the lateral surface of the STG respond more robustly as the hierarchy of linguistic input is increased, that is, phonemes $>$ tones (Crone et al., 2001a), words > phonemes (Flinker et al., 2011) and words > non words (Canolty et al., 2007). One of the striking aspects of high gamma responses is the ability to track activity within a subject, both temporally and spatially on the level of single trials. Cortical activity while listening to words (contrasted with an acoustic control) has been shown to propagate across the STG to the superior temporal sulcus (STS) (Canolty et al., 2007) and when production is required follows to the IFG motor cortices (Brown et al., 2008; Edwards et al., 2010; Flinker et al., 2015; Fukuda et al., 2010; Pei et al., 2011; Towle et al., 2008). Flinker et al., investigated auditory word repetition and found that Broca's area was active as early as 200 ms poststimulus onset and activity was absent by the time the word was articulated (Fig. 43.3). This early pat tern of activity in Broca's area, commencing prior to articulation, and fading by the time of articulation, was evident across a range of tasks including repetition of auditory mono syllabic words, repetition of auditory multisyllabic words and overt word reading (Flinker et al., 2015). Early activity in Broca's area has also been reported during lexical and inflectional processing of words without overt articulation (Sahin et al., 2009). The monosyllabic words in employed by Flinker et al., were comprised of both real words (e.g., hope) as well as pronounceable pseudowords matched for phonotactic probabilities (e.g., yode). Articulation of a novel sequence of phonemes elicited more activity in Broca's area compared with real words but this was not the case in motor cortex. These findings taken together with the early window of activity implicate Broca's area in the formation of an articulatory plan rather than coordination of the articulators themselves. The authors also leveraged the high gamma signals together with Granger causal connectivity analyses to show the directional flow of cortical activity during word repetition. During perception of the auditory word there was a peak of Granger causal flow from STG to Broca's area followed by reciprocal feedback from Broca's area to STG. This feedback influence from Broca's area onto STG was evident until $200 \mathrm{~ms}$ prior to articulation onset and in parallel there was Granger causal flow from Broca's area to motor cortices lasting up to articulation onset as well as feedback from motor cortices onto Broca's area. These temporal dynamics reveal a key role Broca's area plays in manipulating and forwarding cortical representations as a heard word is processed and transformed into an articulatory code. Individual electrodes over the posterior STG (pSTG) have often shown responses to a linguistic unit regardless of input modality when multiple tasks were employed, such as word reading and auditory repetition (Crone et al., 2001b; Flinker et al., 2010) as well as 
(A)
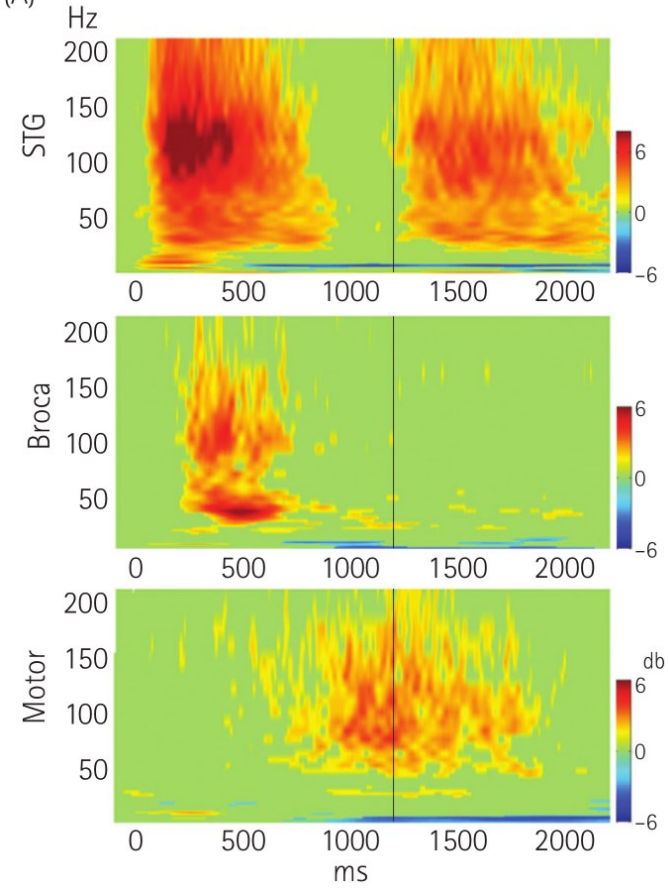

(B)

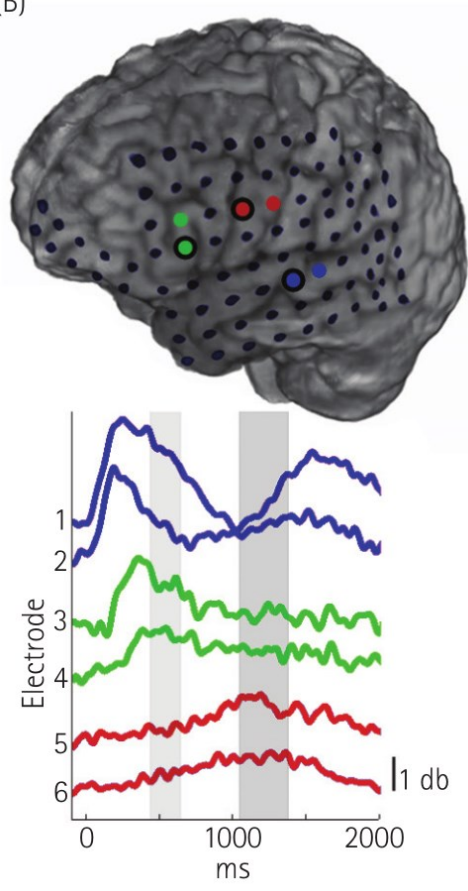

Fig. 43.3 Repetition of monosyllabic words in a representative subject. (A) Event related spectral perturbations (ERSPs), averaged across trials, and locked to the onset of auditory word stimulus. Cortical activation indexed by power increases in high frequencies is first apparent in STG during word perception, subsequently in Broca's area, and finally extends to motor cortex during word production (vertical lines mark mean articulation onset). (B) High frequency power ( $\gamma$ high, $70-150 \mathrm{~Hz}$ ) traces, averaged across trials, and locked to word stimulus onset are shown for STG (blue), Broca (green), and motor (red) electrodes. The first electrode in every pair is marked by a black circle and corresponds to the ERSP plotted on the left. The shaded gray area marks the distribution of articulation onset for this subject (1 SD in each direction).

Adapted from Adeen Flinker, Anna Korzeniewska, Avgusta Y. Shestyuk, Piotr J. Franaszczuk, Nina F. Dronkers, Robert T. Knight, and Nathan E. Crone, Redefining the role of Broca's area in speech, Proceedings of the National

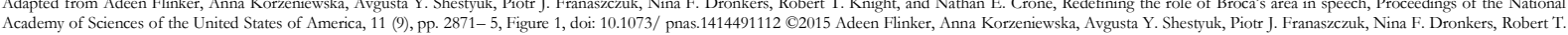
Knight, and Nathan E. Crone. This work is licensed under the Creative Commons Attribution License (CC BY NC ND). It is attributed to the authors

picture naming (Crone et al., 2001b). Similarly, this invariability has been reported across languages in multilingual patients performing a picture naming task where a majority of STG responses were common to both L1 and L2 speech (albeit some sites with L2 specificity) (Cervenka et al., 2011). An interesting finding arising from intracranial research has been the rich spatial variability of responses in STG whether exhibiting modality invariance or selectivity to linguistic hierarchy. Flinker et al. found neighboring sites 4 $\mathrm{mm}$ apart with distinct functional responses: one site responding to both syllables and words and its neighbor selective to words (Flinker et al., 2011). Figure 43.4 shows the distribution of responses over STG in one subject listening to words or phonemes (CV syllables). Similarly, some sites in STG showed a typical suppression during production of speech while neighboring sites showed a selectivity for speech output (Flinker et al., 2010; 2011). While STG reduced activity during speech production has been consistently reported (Crone et al., 2001b; Edwards et al., 2010; Flinker et al., 2010; Fukuda et al., 2010; Pei et al., 2011; Towle et al., 2008), the topography of suppression can vary across the STG during auditory feedback as well as pitch perturbation (Chang et al., 2013; Flinker et al., 2010; Greenlee et al., 2011). This rich topography of responses is sometimes only visible when employing higher density electrode arrays. Chang et al., showed that the spatial topography of pSTG responses encoded the phonetic category of CV syllables rather than 

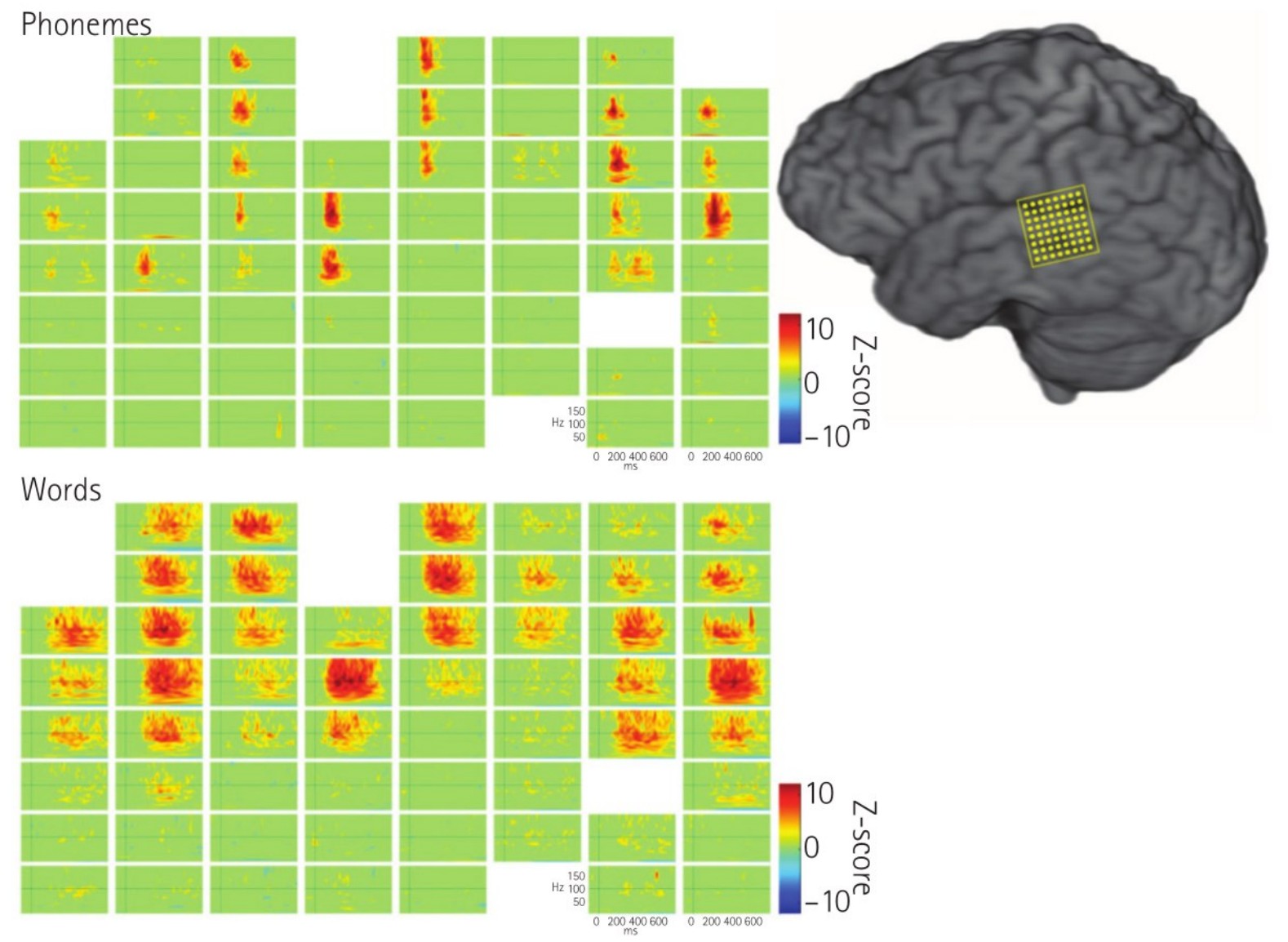

Fig. 43.4 Spatiotemporal responses in a subject listening to phonemes (top) and words (bottoms) during two consecutive recordings across a 64 contact $8 \times 8$ high density electrode grid. ERSPs are shown for each electrode locked to the onset of stimuli. Color scale represents statistically significant changes in power with most activity in the high frequency range (horizontal line in each electrode marks $100 \mathrm{~Hz}$ ). Electrodes with no contact or abnormal signal are not shown.

the acoustic continuum used to synthesize them (Chang et al., 2010). Similarly, Mesgarani et al. used high density ECoG arrays to decode responses during auditory sentences that spanned the English phonetic lexicon. They show that individual electrodes are highly selective to different phonemes and encode acoustic phonetic features. Similarly, high density recordings from Rolandic cortex (pre and postcentral gyri) have shown a somatotopical organization of speech articulators which are phonetically grouped (Bouchard et al., 2013) as well as a surprising acoustic receptive field representation within motor cortex (Cheung et al., 2016). The discovery of the high gamma signal together with leveraging computational approaches has sparked avenues of research that were previously mostly limited to animal research. For example, the spectrotemporal receptive field (STRF) has been tradition ally limited to modeling the relationship between acoustic input and neuronal firing rates. Pasley et al., were the first to use high gamma activity in STRF modeling providing evidence for a robust acoustic representation in STG which consistently reconstructs neural responses based on the acoustic input (Pasley et al., 2012). Similar techniques have been used to elucidate the acoustic phonetic organization in STG (Mesgarani et al., 2014) and how auditory cortex enhances speech intelligibility by rapid tuning of the underlying receptive fields (Holdgraf et al., 2016). In addition to these encoding models (predicting neural activity based on stimulus features) there has been great interest in decoding activity from cortex (predicting a stimulus class based on the neural activity). Pasley et al. provided a reconstruction of the auditory sentences played to the patients based solely on the neural activity in STG (Pasley et al., 2012). Similar approaches are being used to decode imagined speech (Martin et al., 2016a; 2016b) and try to drive a speech prosthesis (Leuthardt et al., 2011). Decoding techniques have also been employed in decoding sensory motor transformations providing evidence that speech transformations occur bilaterally (Cogan et al., 2014). 


\subsection{Future directions}

Intracranial electrophysiology provides an exciting opportunity to explore language processing in the human cortex with high temporal and spatial resolution. While much progress has been made, there still remain many unresolved questions and unaddressed areas in language processing. High gamma has provided an unprecedented index of cortical activity, but its relationship to low frequency and ERP findings in the noninvasive language literature remains understudied. Moreover, a great number of intracranial studies focus on sublexical and lexical processing and there is a lack of research studying higher order linguistic operations. Recent studies have leveraged the high gamma signal in advanced ma chine learning techniques to reconstruct signals based on sets of features. Such approaches are valuable and illuminating but very often are limited to acoustic or sublexical features. For example, acoustic STRF modeling (also known as a type of an encoding model) predicts the neural signal based on the auditory input (e.g., speech) the patient heard. When the model is robust then one can reliably reconstruct the neural signal given a new set of speech stimuli, but such models are limited to the time frequency acoustics of the signal and do not necessarily account for phonetic, sublexical, lexical, semantic, and grammatical structure. That said, such modeling techniques could easily incorporate higher order linguistic structures. Similarly, machine learning decoding techniques that try to predict a target class or variable (e.g., acoustic spectrogram, phoneme, and so on) given a set of neural signals could be augmented to classify lexical, semantic, and syntactic representations. Critically these models have to be driven by and interpreted within current (or novel) theoretical frameworks of language processing. There has been increasingly interest in the role of the medial temporal lobe in language (Covington \& Duff, 2016; Duff \& Brown Schmidt, 2017), partly fueled by recent findings from sEEG research (Hamamé et al., 2014; Jafarpour et al., 2017; Llorens et al., 2016; Piai et al., 2016). The combination of excellent temporal and spatial resolution of iEEG recordings is particularly important for understanding the role of these deeper regions, as they cannot be easily recorded from with scalp based techniques and fMRI approaches are limited in temporal resolution necessary to track the dynamics of speech and language. Future evidence from iEEG recordings will likely greatly expand our understanding of the relations between the language and memory systems. The finding that Broca's area is not involved during articulation per se but rather coordinating articulatory planning is a striking example of the unique advantage intracranial studies provide. Previous non-invasive electrophysiological studies were limited by both the spatial specificity of neural generators as well as motor artifacts during speech production. In contrast, neuroimaging studies of speech production could resolve activity within Broca's area but could not elucidate the exact timing and stage of recruitment. The combined temporal and spatial resolution provided by intracranial recordings, together with minimal contamination from motor artifacts, ideally situates it to elucidate the role of Broca's area during speech production. Nevertheless, such an endeavor requires more than simple speech production tasks such as word repetition and overt reading. Ideally, spontaneous speech and longer, more complex utterances should be investigated. Such an endeavor is not trivial, as it requires novel speech production paradigms that prompt continuous segments of spontaneous speech that are experimentally controlled and are optimally recorded in tandem with neural recordings. One approach that is likely to benefit both speech production research as well as impact patient care is employing decoding and machine learning techniques on large corpora of speech together with the intracranial signals in Broca's area and motor cortices. Such an approach could reveal receptive field properties in frontal cortices while providing a stepping stone toward a neural speech production pros thesis. While the ability to reliably reproduce speech based on intracranial signals is still far in the future, it could provide invaluable insight into the speech production network and its temporal dynamics. 


\section{References}

Adrian, E. D., \& Matthews, B. H. C. (1934). The Berger rhythm: potential changes from the occipital lobes in man. Brain 57:355- 385 .

Allen, E. A., Pasley, B. N., Duong, T., \& Freeman, R. D. (2007). Transcranial magnetic stimulation elicits coupled neural and hemodynamic consequences. Science, 317, 1918- 21.

Ball, T., Kern, M., Mutschler, I., Aertsen, A., \& SchulzeBonhage, A. (2009). Signal quality of simultaneously recorded invasive and noninvasive EEG. NeuroImage, 46, 708- 16.

Belitski, A., Gretton, A., Magri, C., Murayama, Y., Montemurro, M. A., Logothetis, N. K., \& Panzeri, S. (2008). Lowfrequency local field potentials and spikes in primary visual cortex convey independent visual information. Journal of Neuroscience, 28, 5696- 709.

Berger, H. (1929). Über das Elektrenkephalogramm des Menschen. Arcbiv fPsychiatrie, 87, 527- 70.

Berger, H. (1931). Über das Elektrenkephalogramm des Menschen. Archiv fPsychiatrie, 94, 16- 60.

Bouchard, K. E., Mesgarani, N., Johnson, K., \& Chang, E. F. (2013). Functional organization of human sensorimotor cortex for speech articulation. Nature, 495, 327- 32.

Broca, P. (1861). Remarques sur le siege de la faculté du langage articulé, suivies d'une observation d'aphémie (perte de la parole). Bulletins et mémoires de la Société Anatomique de Paris, 36, 330- 56.

Brown, E. C., Rothermel, R., Nishida, M., Juhász, C., Muzik, O., Hoechstetter, K., . . \& Asano, E. (2008). In vivo animation of auditory language induced gamma oscillations in children with intractable focal epilepsy. NeuroImage, 41, 1120-31.

Brugge, J. F., Nourski, K. V., Oya, H., Reale, R. A., Kawasaki, H., Steinschneider, M., \& Howard, M. A. (2009). Coding of repetitive transients by auditory cortex on Heschl's gyrus. Journal of Neurophysiology, 102, $2358-74$.

Buzsáki, G., Anastassiou, C. A., \& Koch, C. (2012). The origin of extracellular fields and currents- EEG, ECoG, LFP and spikes. Nature Reviews Neuroscience, 13, 407- 20.

Buzsáki, G., \& Moser, E. I. (2013). Memory, navigation and theta rhythm in the hippocampal-entorhinal system. Nature Neuroscience, 16, 130- 8.

Canolty, R. T., Soltani, M., Dalal, S. S., Edwards, E., Dronkers, N. F., Nagarajan, S. S., . . \& Knight, R. T. (2007). Spatiotemporal dynamics of word processing in the human brain.Frontiers in Neuroscience, 1, 18596.

Cervenka, M. C., BoatmanReich, D., Ward, J., Franaszczuk, P. J., \& Crone, N. (2011). Language mapping in multilingual patients: Electrocorticography and cortical stimulation during naming. Frontiers in Human Neuroscience, 5, 13.

Chang, E. F., Edwards, E., Nagarajan, S. S., Fogelson, N., Dalal, S. S., Canolty, R. T., . . \& K Knight, R. T. (2011). Cortical spatiotemporal dynamics underlying phonological target detection in humans. Journal of Cognitive Neuroscience, 23, 1437- 46.

Chang, E. F., Niziolek, C. A., Knight, R. T., Nagarajan, S. S., \& Houde, J. F. (2013). Human cortical sensorimotor network underlying feedback control of vocal pitch. Proceedings of the National Academy of Sciences of the United States of America, 110, 2653- 2658.

Chang, E. F., Raygor, K. P., \& Berger, M. S. (2014). Contemporary model of language organization: An overview for neurosurgeons. Journal of Neurosurgery, 122, 250- 61.

Chang, E. F., Rieger, J. W., Johnson, K., Berger, M. S., Barbaro, N. M., \& Knight, R. T. (2010).

Categorical speech representation in human superior temporal gyrus. Nature Neuroscience, 13, 1428- 32. 
Cheung, C., Hamiton, L. S., Johnson, K., \& Chang, E. F. (2016). The auditory representation of speech sounds in human motor cortex. eLife Sciences, 5, e12577.

Cogan, G. B., Thesen, T., Carlson, C., Doyle, W., Devinsky, O., \& Pesaran, B. (2014). Sensory-motor transformations for speech occur bilaterally. Nature, 507, 94- 8 .

Covington, N. V., \& Duff, M. C. (2016). Expanding the language network: Direct contributions from the hippocampus. Trends in Cognitive Sciences, 20, 869- 70.

Crandall, P. H., \& Babb, T. L. (1993). The UCLA Epilepsy Program: historical review 1960-1992. Journal of Clinical Neurophysiology, 10, 226- 38.

Creutzfeldt, O., Ojemann, G., \& Lettich, E. (1989). Neuronal activity in the human lateral temporal lobe. Experimental Brain Research, 77, 451- 75.

Crone, N. E. (2000). Functional mapping with ECoG spectral analysis. Advances in Neurology, 84, 343- 51.

Crone, N. E., Boatman, D., Gordon, B., \& Hao, L. (2001a). Induced electrocorticographic gamma activity during auditory perception. Clinical Neurophysiology, 112, 565- 82.

Crone, N. E., Hao, L., Hart, J., Boatman, D., Lesser, R. P., Irizarry, R., \& Gordon, B. (2001b).

Electrocorticographic gamma activity during word production in spoken and sign language. Neurology, 57, 2045- 53 .

Crone, N. E., Hart, J., Boatman, D., Lesser, R. P., \& Gordon, B. (1994). Regional cortical activation during language and related tasks identified by direct cortical electrical recording. Brain and Language, $47,466-8$.

Crone, N. E., Korzeniewska, A., \& Franaszczuk, P. J. (2011). Cortical $\gamma$ responses: Searching high and low. International Journal of Psychophysiology, 79, 9-15.

Crone, N. E., Miglioretti, D. L., Gordon, B., Lesser, R. P. (1998a). Functional mapping of human sensorimotor cortex with electrocorticographic spectral analysis. II. Event-related synchronization in the gamma band. Brain, 121(Pt 12), 2301- 15.

Crone, N. E., Miglioretti, D. L., Gordon, B., Sieracki, J. M., Wilson, M. T., Uematsu, S., \& Lesser, R. P. (1998b). Functional mapping of human sensorimotor cortex with electrocorticographic spectral analysis. I. Alpha and beta event-related desynchronization. Brain, 121, 2271- 99.

Crone, N. E., Sinai, A., \& Korzeniewska, A. (2006). High frequency gamma oscillations and human brain mapping with electrocorticography. Progress in Brain Research, 159, 275- 95.

Cushing, H. (1909). A note upon the faradic stimulation of the postcentral gyrus in conscious patients.1. Brain, 32, 44- 53.

Duff, M. C., \& BrownSchmidt, S. (2017). Hippocampal contributions to language use and processing. In: Hannula, D. E., \& Duff, M. C. (Eds.), The Hippocampus from Cells to Systems: Structure, Connectivity, and Functional Contributions to Memory and Flexible Cognition (pp. 503- 36). Springer International Publishing, Cham.

Edwards, E. (2005). High gamma activity in response to deviant auditory stimuli recorded directly from human cortex. Journal of Neurophysiology, 94, 4269- 80.

Edwards, E., Nagarajan, S. S., Dalal, S. S., Canolty, R. T., Kirsch, H. E., Barbaro, N. M., \& Knight, R. T. (2010). Spatiotemporal imaging of cortical activation during verb generation and picture naming. NeuroImage, 50, 291- 301.

Feindel, W., Leblanc, R., de Almeida, A. N. (2009). Epilepsy surgery: Historical highlights 1909- 2009. Epilepsia, 50, 131- 51. 
Flinker, A., Chang, E. F., Barbaro, N. M., Berger, M. S., \& Knight, R. T. (2011). Subcentimeter language organization in the human temporal lobe. Brain and Language, 117, 103- 9.

Flinker, A., Chang, E. F., Kirsch, H. E., Barbaro, N. M., Crone, N. E., \& Knight, R. T. (2010). Singletrial speech suppression of auditory cortex activity in humans. Journal of Neuroscience, 30, 16643- 50.

Flinker, A., Korzeniewska, A., Shestyuk, A. Y., Franaszczuk, P. J., Dronkers, N. F., Knight, R. T., \& Crone, N. E. (2015). Redefining the role of Broca's area in speech. Proceedings of the National Academy of Sciences of the United States of America, 112, 2871- 5.

Foerster, O., \& Altenburger, H. (1935). Elektrobiologische Vorgänge an der menschlichen Hirnrinde. Deutsche Zeitschrift $f$ Nervenbeilkunde, 135, 277- 88.

Fried, I., Ojemann, G. A., \& Fetz, E. E. (1981). Languagerelated potentials specific to human language cortex. Science, 212, 353- 6.

Fukuda, M., Rothermel, R., Juhász, C., Nishida, M., Sood, S., \& Asano, E. (2010). Cortical gamma oscillations modulated by listening and overt repetition of phonemes. NeuroImage, 49, 2735- 45.

Gibbs, F. A., Lennox, W. G., Gibbs, E. L. (1936). The electroencephalogram in diagnosis and in localization of epileptic seizures. Archives of Neurology \& Psychiatry, 36, 1225- 35.

Gonzalez Martinez, J., Bulacio, J., Alexopoulos, A., Jehi, L., Bingaman, W., \& Najm, I. (2013).

Stereoelectroencephalography in the "difficult to localize" refractory focal epilepsy: Early experience from a North American epilepsy center. Epilepsia, 54, 323- 30.

Greenlee, J. D. W., Jackson, A. W., Chen, F., Larson, C. R., Oya, H., Kawasaki, H., . . \& Howard, M.

A. III (2011). Human auditory cortical activation during selfvocalization. PLoS One, 6, e14744.

Halgren, E., Kaestner, E., Marinkovic, K., Cash, S. S., Wang, C., Schomer, D. L., . . \& Ulbert, I. (2015). Laminar profile of spontaneous and evoked theta: Rhythmic modulation of cortical processing during word integration. Neuropsychologia, 76, 108- 24.

Halgren, E., Wang, C., Schomer, D. L., Knake, S., Marinkovic, K., Wu, J., \& Ulbert, I. (2006). Processing stages underlying word recognition in the anteroventral temporal lobe. NeuroImage, 30, 1401- 13.

Hamamé, C. M., Alario, F. X., Llorens, A., LiégeoisChauvel, C., TrébuchonDa Fonseca, A. (2014). High frequency gamma activity in the left hippocampus predicts visual object naming performance. Brain and Language, 135, $104-14$.

Hitzig, E. (1900). Hughlings Jackson and the cortical motor centres in the light of physiological research. Brain, 23, 545- 81 .

Holdgraf, C. R., de Heer, W., Pasley, B., Rieger, J., Crone, N., Lin, J. J., . . \& Theunissen, F. E. (2016). Rapid tuning shifts in human auditory cortex enhance speech intelligibility. Nature Communications, 7, 13654 .

Horsley, V. (1909). The Linacre Lecture on the function of the socalled motor area of the brain: Delivered to the Master and Fellows of St. John's College, Cambridge, May 6, 1909. British Medical Journal, 2, 121- 32.

Howard, M. A. III, Volkov, I. O., Abbas, P. J., Damasio, H., Ollendieck, M. C., \& Granner, M. A. (1996a). A chronic microelectrode investigation of the tonotopic organization of human auditory cortex. Brain Research, 724, 260- 4 .

Howard, M. A., Volkov, I. O., Granner, M. A., Damasio, H. M., Ollendieck, M. C., \& Bakken, H. E. (1996b). A hybrid clinicalresearch depth electrode for acute and chronic in vivo microelectrode recording of human brain neurons. Technical note. Journal of Neurosurgery, 84, 129- 32.

Jacobs J, Manning JR, Kahana MJ (2010). Response to Miller: "Broadband" vs. "high gamma" electrocorticographic signals. Journal of Neuroscience, 30(19). 
Jafarpour, A., Piai, V., Lin, J. J., \& Knight, R. T. (2017). Human hippocampal preactivation predicts behavior. Scientific Reports, 7, 5959.

Jasper, H., \& Kershman, J. (1941). Electroencephalographic classification of the epilepsies. Archives of Neurology and Psychiatry, 45, 903- 43.

Jasper, H., Pertuisset, B., \& Flanigin, H. (1951). EEG and cortical electrograms in patients with temporal lobe seizures. AMA Archives of Neurology and Psycbiatry, 65, 272- 90.

Jerbi, K., Freyermuth, S., Dalal, S., Kahane, P., Bertrand, O., Berthoz, A., \& Lachaux, J. P. (2009). Saccade related gammaband activity in intracerebral EEG: Dissociating neural from ocular muscle activity. Brain Topography, 22, 18-23.

Kajikawa, Y., \& Schroeder, C. E. (2011). How local is the local field potential? Neuron, 72, 847- 58.

Kovach, C. K., Tsuchiya, N., Kawasaki, H., Oya, H., Howard, M. A. III, \& Adolphs, R. (2011).

Manifestation of ocularmuscle EMG contamination in human intracranial recordings. NeuroImage, 54, 213- 33 .

Krause, F., \& Thorek, M. (1912). Surgery of the brain and spinal cord: based on personal experiences. (Haubold, A. and Thorek, M., Trans). Rebman, New York, NY.

Lachaux, J. P., Rudrauf, D., \& Kahane, P. (2003). Intracranial EEG and human brain mapping. Journal of Physiology Paris, 97, 613-28.

Lega, B. C., Jacobs, J., \& Kahana, M. (2012). Human hippocampal theta oscillations and the formation of episodic memories. Hippocampus, 22, 748- 61.

Leuthardt, E. C., Gaona, C., Sharma, M., Szrama, N., Roland, J., Freudenberg, Z., . . \& Schalk G (2011). Using the electrocorticographic speech network to control a braincomputer interface in humans. Journal of Neural Engineering, 8, 036004.

LiégeoisChauvel, C., de Graaf, J. B., Laguitton, V., \& Chauvel, P. (1999). Specialization of left auditory cortex for speech perception in man depends on temporal coding. Cerebral Cortex, 9, 484-96.

LiégeoisChauvel, C., Lorenzi, C., Trébuchon, A., Régis, J., \& Chauvel, P. (2004). Temporal envelope processing in the human left and right auditory cortices. Cerebral Cortex, 14, 731- 40.

LiégeoisChauvel, C., Musolino, A., \& Chauvel, P. (1991). Localization of the primary auditory area in man. Brain, 114(Pt 1A), 139- 51.

Llorens, A., Dubarry, A.S., Trébuchon, A., Chauvel, P., Alario, F.X., \& LiégeoisChauvel, C. (2016). Contextual modulation of hippocampal activity during picture naming. Brain and Language, 159, 92- 101.

Llorens, A., Trébuchon, A., LiégeoisChauvel, C., \& Alario, F. X. (2011). Intracranial recordings of brain activity during language production. Frontiers in Psychology, 2, 375.

Lüders, H. O. (2008). Textbook of Epilepsy Surgery. Informa, London.

Martin, S., Brunner, P., Iturrate, I., del R. Millán, J., Schalk, G., Knight, R. T., \& Pasley, B. N. (2016a). Word pair classification during imagined speech using direct brain recordings. Scientific Reports, 6, 25803.

Martin, S., Millán, J. D. R., Knight, R. T., \& Pasley, B. N. (2016b). The use of intracranial recordings to decode human language: Challenges and opportunities. Brain and Language. doi: 10.1016/

j.bandl.2016.06.003

McCarthy, G., Nobre, A. C., Bentin, S., \& Spencer, D. D. (1995). Languagerelated field potentials in the anteriormedial temporal lobe: I. Intracranial distribution and neural generators. Journal of Neuroscience, 15 , 1080- 9 . 
Mesgarani, N., Cheung, C., Johnson, K., \& Chang, E. F. (2014). Phonetic feature encoding in human superior temporal gyrus. Science (New York, NY), 343, 1006- 10.

Meyer, P., Mecklinger, A., Grunwald, T., Fell, J., Elger, C. E., \& Friederici, A. D. (2005). Language processing within the human medial temporal lobe. Hippocampus, 15, 451-9.

Miller, K. J. (2010). Broadband spectral change: Evidence for a macroscale correlate of population firing rate? Journal of Neuroscience, 30, 6477-9.

Mukamel, R., Gelbard, H., Arieli, A., Hasson, U., Fried, I., \& Malach, R. (2005). Coupling between neuronal firing, field potentials, and fMRI in human auditory cortex. Science, 309, 951- 4.

Mullin, J. P., Sexton, D., AlOmar, S., Bingaman, W., \& Gonzalez Martinez, J. (2016). Outcomes of subdural grid electrode monitoring in the stereoelectroencephalography era. World Neurosurgery, 89, 255- 8.

Nir, Y., Fisch, L., Mukamel, R., GelbardSagiv, H., Arieli, A., Fried, I., \& Malach, R. (2007). Coupling between neuronal firing rate, gamma LFP, and BOLD fMRI is related to interneuronal correlations. Current Biology, 17, 1275- 85.

Nobre, A. C., \& McCarthy, G. (1995). Languagerelated field potentials in the anteriormedial temporal lobe: II. Effects of word type and semantic priming. Journal of Neuroscience, 15, 1090- 8.

Nourski, K. V., Reale, R. A., Oya, H., Kawasaki, H., Kovach, C. K., Chen, H., . . \& Brugge, J. F. (2009). Temporal envelope of timecompressed speech represented in the human auditory cortex. Journal of Neuroscience, 29, 15564- 74.

Ojemann, G., Ojemann, J., Lettich, E., \& Berger, M. (1989). Cortical language localization in left, dominant hemisphere: an electrical stimulation mapping investigation in 117 patients. Journal of Neurosurgery, 71, 316- 26.

Ojemann GA (1987). Surgical therapy for medically intractable epilepsy. Journal of Nenoscience, 66, 489- 99.

Pasley, B. N., David, S. V., Mesgarani, N., Flinker, A., Shamma, S. A., Crone, N. E., . . \& Chang, E. F. (2012). Reconstructing speech from human auditory cortex. PloS Biol, 10, e1001251.

Pei, X., Leuthardt, E. C., Gaona, C. M., Brunner, P., Wolpaw, J. R., Schalk, G. (2011). Spatiotemporal dynamics of electrocorticographic high gamma activity during overt and covert word repetition.

NeuroImage, 54, 2960- 72 .

Penfield, W., \& Boldrey, E. (1937). Somatic motor and sensory representation in the cerebral cortex of man as studied by electrical stimulation. Brain, 60, 389- 443 .

Penfield, W., \& Erickson, T. C. (1941). Epilepsy and Cerebral Localization. Charles C. Thomas, Oxford. Penfield, W., \& Roberts, L. (1959). Speech and Brain Mechanisms. Princeton University Press, Princeton, NJ.

Piai, V., Anderson, K. L., Lin, J. J., Dewar, C., Parvizi, J., Dronkers, N. F., \& Knight, R. T. (2016). Direct brain recordings reveal hippocampal rhythm underpinnings of language processing. Proceedings of the National Academy of Sciences of the United States of America, 113, 11366-71. doi: 10.1073/pnas.1603312113

Ray, S., Hsiao, S. S., Crone, N. E., Franaszczuk, P. J., \& Niebur, E. (2008). Effect of stimulus intensity on the spikelocal field potential relationship in the secondary somatosensory cortex. Journal of Neuroscience, 28, $7334-43$.

Risinger, M. W., \& Gumnit, R. J. (1995). Intracranial electrophysiologic studies. Neuroimaging Clinics of North America, 5, 559- 73.

Rutishauser, U., Ross, I. B., Mamelak, A. N., \& Schuman, E. M. (2010). Human memory strength is predicted by theta frequency phaselocking of single neurons. Nature, 464, 903- 7 . 
Sahin, N. T., Pinker, S., Cash, S. S., Schomer, D., \& Halgren, E. (2009). Sequential processing of lexical, grammatical, and phonological information within Broca's area. Science, 326, 445- 9.

Sanai, N., Mirzadeh, Z., \& Berger, M. S. (2008). Functional outcome after language mapping for glioma resection. New England Journal of Medicine, 358, 18- 27.

Talairach, J., David, M., \& Tournoux, P. (1958). L'exploration chirurgicale stéréotaxique du lobe temporal dans l'épilepsie temporale: Repérage anatomique stéréotaxique et technique chirurgicale. Masson, Paris.

Towle, V. L., Yoon, H. A., Castelle, M., Edgar, J. C., Biassou, N. M., Frim, D. M., . . \& \& Kohrman, M. H. (2008). ECoG gamma activity during a language task: differentiating expressive and receptive speech areas. Brain, 131, 2013-27.

Wellmer, J., Groeben, von der F., Klarmann, U., Weber, C., Elger, C. E., Urbach, H., . . \& Lehe, von M. (2012). Risks and benefits of invasive epilepsy surgery workup with implanted subdural and depth electrodes. Epilepsia, 53, 1322- 32.

Wernicke, C. (1874). Symptomenkomplex: Eine psychologische Studie auf anatomischer Basis. Cohn und Weigert, Breslau.

WiddessWalsh, P., Jeha, L., Nair, D., Kotagal, P., Bingaman, W., Najm, I. (2007). Subdural electrode analysis in focal cortical dysplasia: predictors of surgical outcome. Neurology, 69, 660- 7.

Wieser, H. G., \& Elger, C. E. (2012). Presurgical Evaluation of Epileptics: Basics, Techniques, Implications. SpringerVerlag, Berlin. 